\title{
Design of the Motion Control System for a Long Endurance AUV
}

\author{
CAO Jian ${ }^{1}$, ZHAO Shiqi $^{2}$, ZHANG Lei $^{1, a}$, ZHUANG Jiayuan ${ }^{1}$, ,BI Xiaosheng ${ }^{1}$ \\ ${ }^{1}$ Science and Technology on Underwater Vehicle Laboratory, Harbin Engineering University, \\ Nantong Street 145, Heilongjiang, China \\ ${ }^{2}$ China State Shipbuilding Corporation, Zhuyuguoji Buliding One, Shouti South Street NO.9, \\ Haidian , Beijing, China \\ acheung103@163.com
}

\begin{abstract}
Keywords: Autonomous Underwater Vehicle, Long Endurance, Motion Control, System Architecture

Abstract. The long endurance Autonomous Underwater Vehicle (AUV) could finish autonomous mission process without any human aids in a long time. As an artificial intelligence system, AUV has high independence, reliability and adaptability to ocean environment. An efficient architecture of AUV plays an important roll in achieving those properties. A long endurance AUV, which is used for ocean exploring, was introduced. And its motion control system architecture was described. The architecture is divided into four parts including blackboard system, elementary behavior agent group, reflection behavior agent group and execution agent. Both the hardware and software in the control system were described. Finally, the experiments in tank and those at sea were conducted to test the architecture and the methods of the control system, and the results proved that the control system of the AUV is reliable, flexible and extensible.
\end{abstract}

\section{Depiction of "LE-AUV"}

The research on long endurance AUV was initially carried out in the United States and other developed countries[1-3]. "LE-AUV" is designed as a long endurance AUV for integrated ocean exploration. It can be used to survey submarine terrain, detect and identify underwater objects, detect ocean oil-gas pipelines, acquire ocean environment data, detect and position submarine communication optical cables.

"LE-AUV" can be divided into 8 subsystems according to the system function and mission requirement, such as basic control system, underwater navigation system, communication system, system of object detecting and environment data acquisition, execution system, safety checking system, self-rescue system and power system.

The basic control system includes software and hardware about intelligent reasoning method and control strategy. The navigation system contains undersized inertial navigation system, Doppler velocity log, depth meter, altimeter and GPS. The communication system includes hydroacoustic communication system, radio communication equipment, and fiber communication device (used for system debugging). The system of object detecting and environment data acquisition consists of forward-looking sonar, low-light level camera, side-scan sonar, conductivity-temperature-depth sensor, and magnetic detector. The execution system includes rudders and thrusters. There are two ducted propellers used as main thrusters which are set at the stern, and each of them can provide the maximal force of $110 \mathrm{~kg}$. There are two tunnel propellers used as vertical thrusters which are set respectively at the bow and the stern, and each of them can provide the maximal force of $25 \mathrm{~kg}$. There is a pair of elevating rudders which are set at the stern. Besides there is a rudder set at the stern to control heading. The safety checking system contains leaking detector, hydrogen detector, and battery state monitor system. The self-rescue system has an individual power supply, and it will automatically start when the depth value is abnormal to keep the AUV safe. The power system is formed by charged Li-ion battery packs which are laid in two individual watertight cabins 


\section{Motion Control System Architecture Based on Agent}

System architecture was researched to construct a type of mechanism to make the modules mentioned above work reasonably in space and time area, thus the information data can flow successfully in the whole system[4]. The motion control architecture was constructed based on both behaviorism method and agent technology [5]. The agent interacts with the environment via the communicator based on explicit mode and implicit mode. The environment could be social environment which is social network consisting of several agents, and it could also be physical environment, such as sensor devices, actuators and so on. The implicit communication needs to check the changes of the environment made by the other agent. And the explicit communication is the direct exchange of the information between two agents. The interpreter processes the input information and identifies the character state to form the environment information described by internal language[6]. In accordance with the information, the executor reacts based on certain method, and the result will feedback to the environment via the communicator.

The motion control architecture was divided into four parts including blackboard system, elementary behavior agent group, reflection behavior agent group and execution agent, shown in Fig.1. The blackboard system is not only information processing and management center, but also agents' behavior control center. As the executable unit of motion controller, elementary behavior agent group makes AUV achieve three kinds of motion including surge, yaw and heave by certain control algorithm[7]. Reflection behavior agent group is the unit by which the behavior of AUV can be achieved in another way, and it works when the system has fault. Execution agent finally drives the actuators of the system.

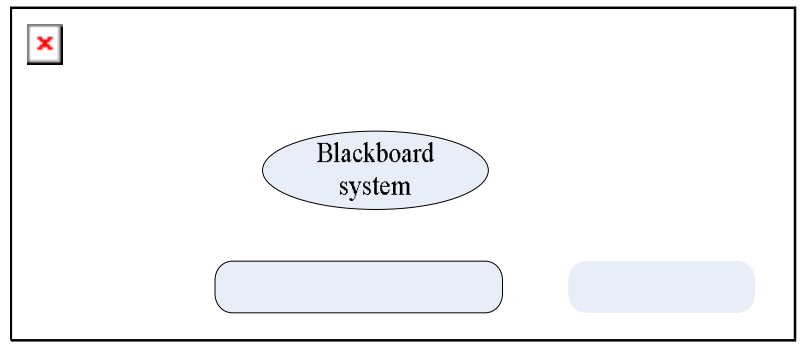

Fig.1 Motion control system architecture of "LE-AUV"

\section{Hardware and Software of Embedded Motion Control System}

\section{Hardware of Embedded Motion Control System}

The hardware of motion control system of "LE-AUV", shown in Fig.2, was constructed with multiboard embedded systems based on PC104 bus, which consist of a core module with an integrated Intel Celeron CPU, an 8-channel serial port module, a DIO module based on PC104 bus and a power module providing $5 \mathrm{~V}$ voltage, besides. There are some other kinds of boards related with the external devices.
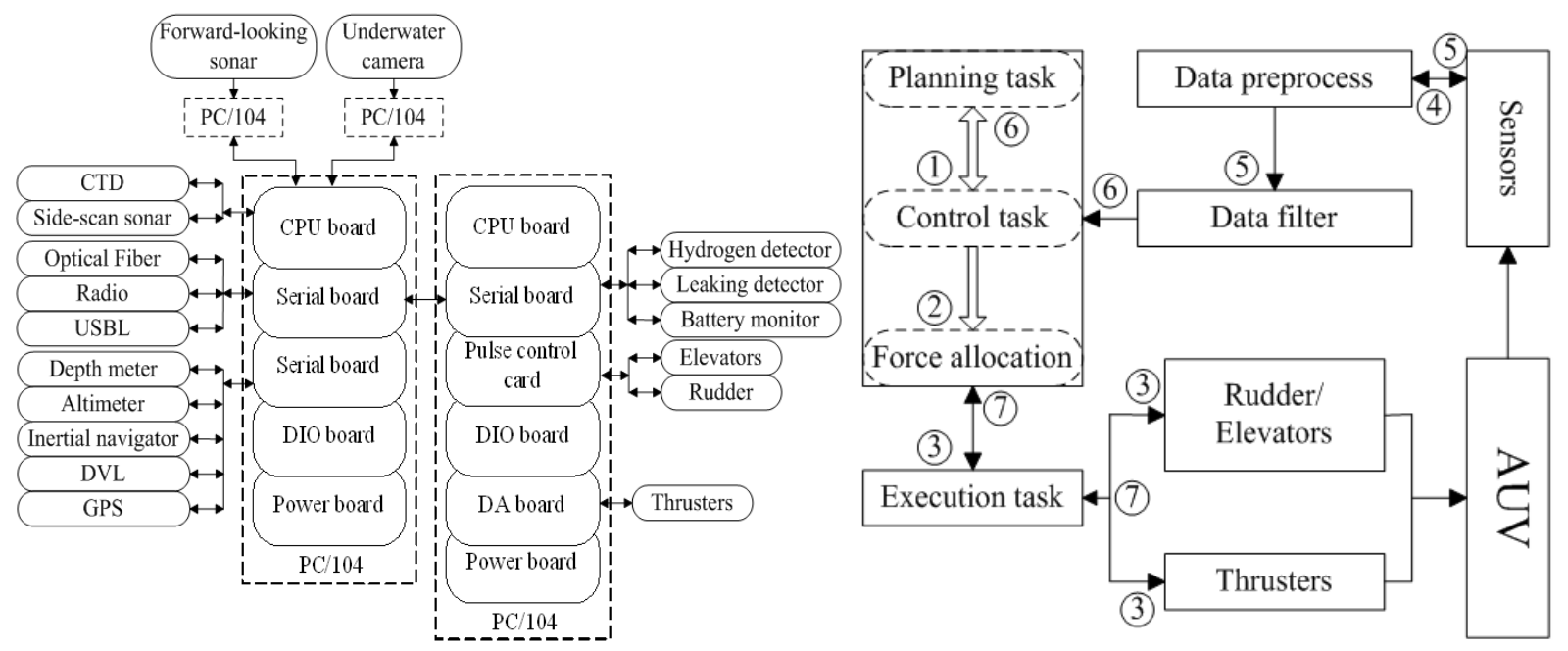

Fig.2 Motion control system Structure

Fig.3 Data flow of embedded motion control system 
The core module uses CMX147786CX CPU board made by RTD Company, which integrates an Intel Celeron processor with $650 \mathrm{MHz}$ main frequency, an AGP S3 Savage SVGA imagine processor, 512MB memory, a 4GB DOA electronic disk, a 6-channel DMA, a 15-channel interrupt, 3 counters, and an APIC.

The serial port board uses EMERALD-MM-8 made by DIAMOND Company, which ingrates a 8-channel serial port supporting RS-232/RS-422/RS-485, and 16C654UART supporting a max speed $460.8 \mathrm{kbps}$.

Digital IO module (namely DIO board) uses DM6856 made by RTD Company, which integrates independently controlled 16-channel digital output with photoelectric isolation, and 16-channel digital input with photoelectric isolation. This board is used as a switch of the sensors.

Power module uses XPWR104HR power board made by RTD Company with 8-32V DC as input and $5 \mathrm{~V}$ or $12 \mathrm{~V}$ DC as output.

\section{Software of Embedded Motion Control System}

The software of motion control system works in the embedded real-time OS, vxWorks [8]. The software data flow chart of "LE-AUV" was shown in Fig.3. $\square$ represents the instructions of planning system. $\square$ indicates the motion control system output. $\square$ represents the execution instructions sending from force allocation. $\square$ represents the original data from sensors. $\square$ indicates the pre-processed sensor data, it can be transmitted to data filter module, meanwhile, it can also be input to a certain sensor as reference data. $\square$ indicates the data generated by data filter module, it is the input of motion control system as well as $\square$. $\square$ represents the feedback data from actuators.

VxWorks is an embedded real-time operation system with priority-based preemptive multitask scheduling, and the function of motion control system is achieved in vxWorks by multitask scheduling, thus it is very important to assign the tasks in software architecture. When the motion control system runs, its tasks can be divided into 5 types:

a) Receiving original data. It includes the acquirement of the original data of environment perception system and motion perception system. The data, mainly acquired via serial ports, includes data of depth indicator, height indicator, DVL, inertia navigator, and the state information of actuators, the voltage information of batteries, the leaking information.

b) Data acquirement and process of planning instruction. Data process means resolving, preprocess (wild-point elimination and data fusion) and smooth filtering of original data. Since the original data received from external devices has particular format which cannot be used directly, it needs to be transmitted to the format that can be accepted by motion controller. The planning instructions can be easily received by memory share.

c) Solving motion control instruction. It includes control algorithm and force allocation. The inputs of controller are processed sensor data and target state data out of planning system, the outputs are the required forces. Force allocation module can transmit the required forces to the voltage of a certain thruster or the angle of a certain rudder.

d) Data and instruction output. Data output mainly means dispatching some data to a certain sensor as reference information and sending AUV state information and the environment information to planning system. Instruction output means sending to execution task the voltages and angles received from force allocation module.

e) Emergency handling. If certain fault happens to the sensor, thruster or rudder during work, the system can handle the situation according to fault level.

\section{Experiments}

In order to test the motion control system mentioned above, semi-physical simulation experiment, tank experiment and sea experiment have been conducted in sequence.

Fig. 4 and Fig. 5 show the result of the pipe tracking test conducted in tank. Fig. 4 shows the image of the pipe laid on the bottom of the tank. Fig.5 shows the trace of "LE-AUV" during the test, and the trace is quite coincident with the pipe. 


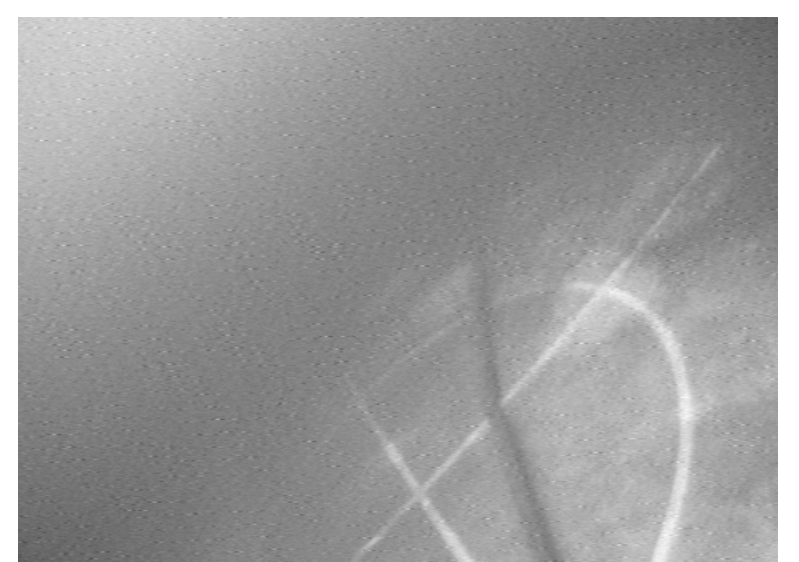

Fig.4 Image of the pipe acquired by "LE-AUV"

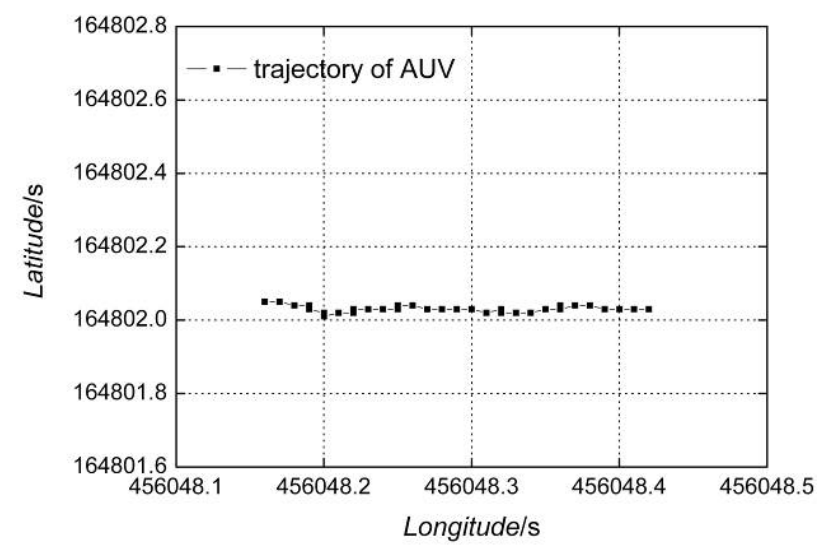

Fig.5 Trace in pipe racking test

Fig. 6 and Fig.7 show the result of sea experiment conducted in the sea nearby Penglai. Fig.6 shows the basic motion control result. Fig.7 shows the voyage trace of "LE-AUV" during the experiment.
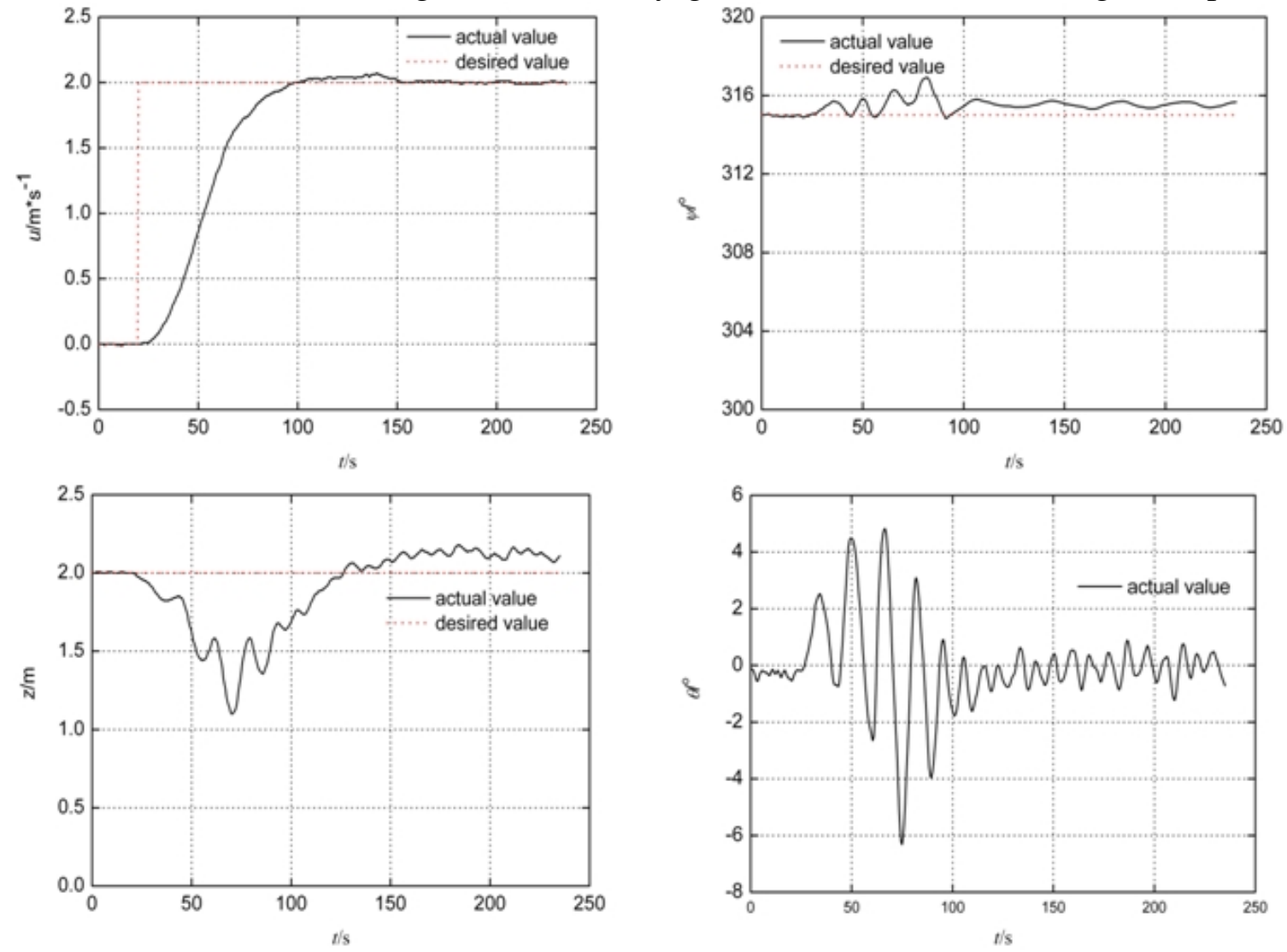

Fig.6 Result of motion control

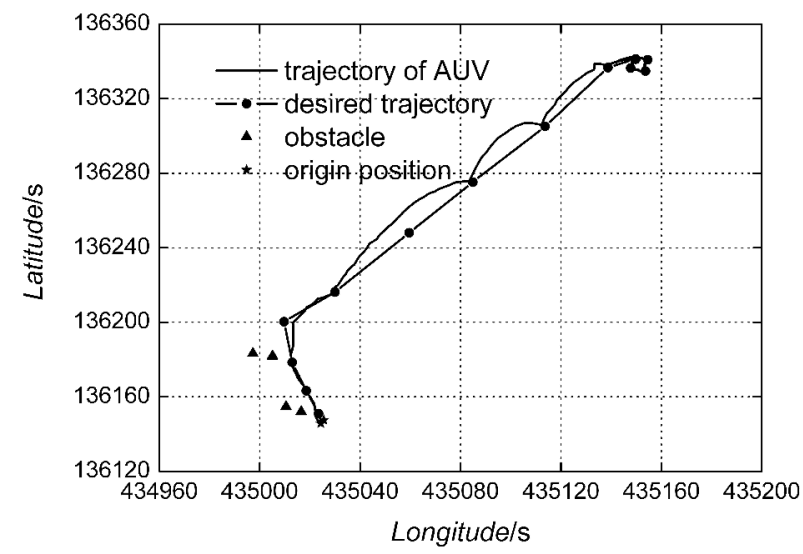

Fig.7 Voyage trace of "LE-AUV" 


\section{Conclusions}

The successful application to integrated ocean exploration AUV shows that the agent-based motion control system proposed in this paper has good robustness, flexibility and expansibility. And it can be used as a reference to other complex intelligent system.

The whole motion control system is implemented based on a famous embedded real-time operation system, vxWorks, and the detail of the software consists of many tasks, thus the division and scheduling of the system task is very important.

\section{Acknowledgments}

This work was supported by Postdoctoral Science Foundation of China (2014M561333), National Natural Science Foundation of China (Projects: 51609047, 51509054 and 51409054).

\section{References}

[1] Oceanus magazine online, "Nereus Soars to the Ocean's Deepest Trench", Massachusetts, United States: Woods Hole Oceanographic Institution (Online edition, 2009).

[2] ECA Robotics, "ALISTER - Autonomous Underwater Vehicle", Toulon Cedex, France: ECA Inc. (2001).

[3] Von alt C.. "REMUS 100 transportable mine countermeasure package", OCEANS 2003 Proceedings, Vol. 4, pp. 1925-1930(2003).

[4] P. Ridao, and J. Batlle, "O2CA2, a new object oriented control architecture for autonomy: the reactive layer", Control Engineering Practice, Vol. 10, No. 8, pp. 857-873 (2002).

[5] Z. Lei, P. Yongjie, S. Yumin, L. Yannan, "HPSO-based fuzzy neural network control for AUV", Journal of Control Theory and Application, Vol. 6, No. 3, pp. 322-326,(2008).

[6] M. Larry, "Frontiers in seafloor mapping and visualization", Marine Geophysical Researches, Vol. 27, No. 1, pp. 7-17(2006).

[7] B. Xinqian, Q. Zheng, Y. Zheping, "Design and evaluation of a hierarchical control architecture for an autonomous underwater vehicle", Journal of Marine Science and Application, Vol. 7, No. 1 , pp.53-58(2008).

[8] WindRiver Inc, "Tornado User Guide", USA: WindRiver Inc.( 2002). 